\title{
NATURAL CONVECTION FOR ANOMALOUS DENSITY VARIATION OF WATER : NUMERICAL BENCHMARK
}

\author{
Tomasz Michałek $^{*}$, Tomasz A. Kowalewski ${ }^{*}$, Bozidar Šarler ${ }^{* *}$ \\ *Polish Academy of Sciences IPPT PAN, Swietokrzyska 21, PL 00-049 Warszawa, Poland \\ ** Nova Gorica Polytechnic, Laboratory for Multiphase Processes, SI-5000 Nova Gorica, \\ Vipavska 13, Slovenia
}

Summary: A steady state natural convection in a differentially heated cavity for temperatures in a vicinity of the freezing point is used to investigate and compare performance of four different numerical methods: finite differences, finite volume, finite elements and mesh-free diffuse approximation method. A primary aim of the exercise is to define a new numerical benchmark solution for natural convection problems, which includes challenging configuration of strongly non-linear buoyancy term. This configuration is used to test performance of two popular commercial codes (Fluent and Fidap) and to compare them with two "classical" finite difference codes and the new promising mesh-free implementation.

\section{PROBLEM FORMULATION}

The accurate solution of natural convection flow in enclosures is a crucial task in a goal of achieving precise modelling of solidification problems. Serious discrepancies between numerical and experimental results encountered for a simple problem of ice formation in a differentially heated cavity [1] motivated us to revise reliability and performance of typical solvers used form simulating heat transfer phenomena. Traditionally such solvers are verified using reference solution defined by Graham de Vahl Davis [2] over 20 years ago. It describes steady state natural convection of a low Prandtl number $(\operatorname{Pr}=0.71)$, Boussinesq fluid in a differentially heated square cavity, with two isothermal and two adiabatic walls. It appears that this largely idealized configuration does not permit to depict bad performing schemes, especially for strong non-linear variation of the fluid density function.

The aim of this work is to formulate a new benchmark solution for verification of numerical codes employed for modelling ice formation problems. The proposed benchmark configuration concerns steady-state natural convection of water in the differentially heated square cavity. By setting the temperature range of isothermal walls close to the freezing point $\left(\mathrm{T}_{\mathrm{H}}=10^{\circ} \mathrm{C}, \mathrm{T}_{\mathrm{C}}=0^{\circ} \mathrm{C}\right)$ and by adopting non-linear variation of the water density with temperature a challenging flow configuration with two counter-rotating re-circulation zones is obtained. The competing effects of positive and negative buoyancy force, and interacting layers of hot and cold liquid, create interesting and difficult to model flow pattern. The two circulations create nearly vertical stratification, separating fluid in the cavity into two regions separated by the density maximum. An interesting configuration for studying heat transfer in a thin, well defined mixing zone separating hot and cold region of the same fluid is obtained. The Rayleigh (Ra) and Prandtl (Pr) numbers describing investigated configuration are based on the fluid properties taken at the reference temperature and the cavity height $(\mathrm{L}=0.038 \mathrm{~m})$. Their values are $\mathrm{Ra}=1.50310^{6}, \mathrm{Pr}=13.31$. This configuration was employed in our previous studies of freezing of water [1].

Details of the benchmark solution are given elsewhere [3]. In the following, we present numerical results obtained for this configuration with two commercial codes, finite volume code Fluent (FLU) and finite element code Fidap (FID) [4], and compare them with the reference finite difference approximation code FRECON3V [5], being a revised, variable properties version of the classical FRECON (FRE) [2]. Finally performance of the new mesh-free numerical approach based on diffuse approximation method [6,7] is investigated and compared with the defined benchmark solutions. For sake of completeness of the most frequently used numerical methodologies, the benchmark is also used to verify performance of a second-order, finite difference, vorticity - streamfunction code SOLVSTR (STR).

\section{NUMERCIAL ALGORITHMS AND SELCTION OF REFERENCE SOLUTIONS}

Extensive mesh-sensitivity tests were performed for each of the five investigated codes and the result of the best performing algorithm are selected as a reference solution. Usual practice of comparing values of flow field parameters at few selected points is not adequate for verifying ability of a code to reproduce fine details of the flow structure. Our tests revealed that even small deviations (2\% - 5\%) from the reference solution, usually reported as reasonable or even excellent agreement, correspond to distinct changes of the flow pattern. Such changes become responsible for differences in the local mass and heat transfer in the system, and are not tolerable when phase change processes are present (e.g. freezing of water).

Hence, to obtain better insight into differences or similarities of the flow structures obtained from the different solvers, the second step of the verification procedure is proposed. It is based on calculating deviation of the velocity profiles extracted along three selected lines: horizontal and vertical centrelines, and the vertical line passing through the mixing zone at the cold wall $(\mathrm{x}=0.9)$. The mesh sensitivity analysis performed for five investigated codes led us to select as a reference, flow field obtained for the finite difference code FRECON3V on the uniform $201 \times 201$ mesh. The velocity and temperature profiles extracted along above mentioned lines are approximated with the high order polynomial and treated as a benchmark solution. The numerical values of the polynomial coefficients obtained permit to perform standard error analysis. An assessment on the accuracy of the solution is obtained calculating relative differences in terms of defined below standard deviations $\sigma$, evaluated for the polynomials describing benchmark profiles and corresponding values extracted from the interrogated solution. Nine indicators are defined and used to evaluate accuracy of the solutions: $\sigma_{\mathrm{u} 1}, \sigma_{\mathrm{w} 1}, \sigma_{\mathrm{t} 1}, \sigma_{\mathrm{u} 2}, \sigma_{\mathrm{w} 2}, \sigma_{\mathrm{t} 2}, \sigma_{\mathrm{u} 3}, \sigma_{\mathrm{w} 3}$ and $\sigma_{\mathrm{t} 3}$. They describe standard deviations calculated for two 
velocity components $U, W$ and temperature $T$ for profiles extracted at centre-lines $\mathrm{Y}=0.5, \mathrm{X}=0.5$, and vertical line $\mathrm{X}=0.9$.

The proposed accuracy indicators can be easily used to assess performance of any numerical solutions, regardless of dimension of the mesh size, as well as to estimate the rate of convergence of successive solutions. Comparison of all tested algorithms was done. Figures 1 and 2 show mesh dependence of $\sigma_{\mathrm{u} 1}, \sigma_{\mathrm{w} 1}$ for $\mathrm{U}, \mathrm{W}$ velocity profiles at $\mathrm{Y}=0.5$ and $\sigma_{\mathrm{u} 2}, \sigma_{\mathrm{w} 2}$ for $\mathrm{U}, \mathrm{W}$ velocity profiles at $\mathrm{X}=0.5$, respectively.

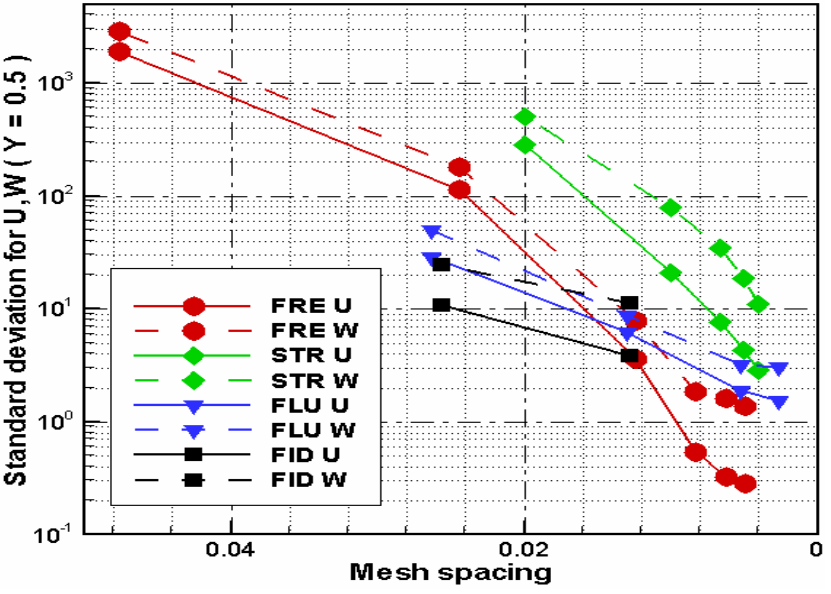

Figure 1

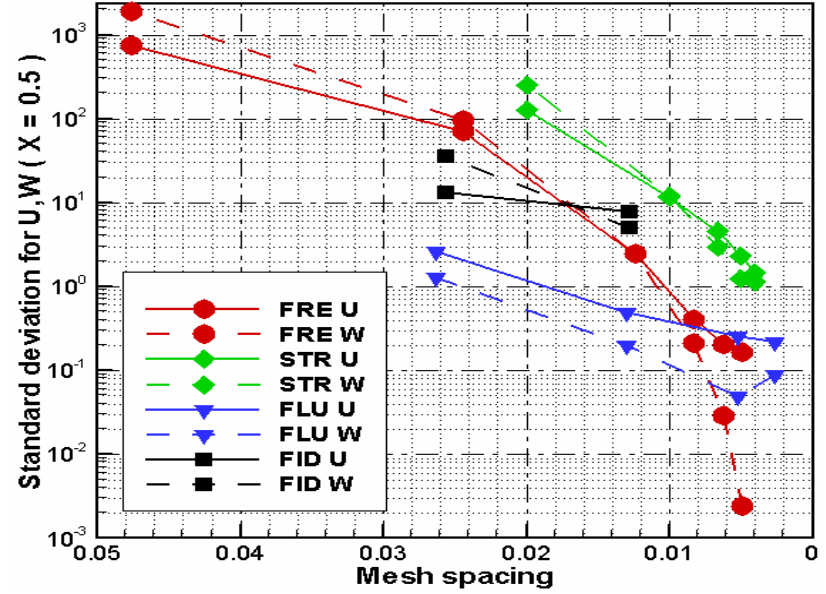

Figure 2

The value of $\sigma_{\mathrm{u} 1}, \sigma_{\mathrm{w} 1}, \sigma_{\mathrm{u} 2} \sigma_{\mathrm{w} 2}, \sigma_{\mathrm{u} 3}, \sigma_{\mathrm{w} 3}, \sigma_{\mathrm{t} 1}, \sigma_{\mathrm{t} 2}, \sigma_{\mathrm{t} 3}$ for the finest mesh FRECON3V (red circle in Figure 1 and 2 ) was taken as a reference error indicator. Generously setting cut-off value for standard deviation equal 3 we are able in clear and straightforward manner decide whether an interrogated solution is close enough to the reference solution to be accepted.

\section{CONCLUSIONS}

New reference solution for testing numerical codes dealing with the natural convection is proposed. The solution is obtained for very fine mesh and used for competition with four other codes. Nine velocity profiles are extracted and given in form of high order polynomials. The measure of performance of the solutions in question is investigated in terms of the standard deviation of the corresponding velocity profiles.

Two commercial codes are verified using above method, along with a new mesh-free numerical approach as well as the classical finite difference approximation code. It appears that the defined measure of the code accuracy is very sensitive, allowing to detect minute deviations occurring in the velocity profiles. The precise analysis of defined convergence indicators allowed to ascertain that among tested four codes, only finest Fluent solution (blue triangle in Figure 1 and 2) was close enough to the reference solution to be acceptable. It is noteworthy that the Fluent solution was obtained on the mesh of $380 \times 380$ nodes, almost twice as dense as the reference solution. All other solutions even obtained for very fine meshes, appeared to be inaccurate, according to the defined measure.

The new, promising approach based on diffuse approximation in mesh-free method turned out to perform intolerable slow in comparison with classical method. Obviously due to the simple geometry we could not observe any advantages of mesh-free approach. The aim of this comparison was solely verification of performance of the method applied to natural convection problem. Future work is necessary to improve initial phase of this methodology, including application of randomly generated collocation points or its generation using predefined measure of sensitivity as well as application of more sophisticated solvers.

\section{References}

[1] T.A. Kowalewski, M. Rebow "Freezing of water in a Differentially Heated Cubic Cavity", Int. J. of Comp. Fluid Dyn, 11:193-210, 1999.

[2] G. de Vahl Davis "Natural convection of air in a square cavity: a bench mark numerical solution" Int. Journal for Numerical Methods in Fluids, 3:249-264, 1983

[3] T. Michalek, T. Kowalewski, B. Sarler „Natural Convection for Anomalous Density Variation of Water : Numerical Benchmark", Progress in Computational Fluid Dynamics, 2004 (in print)

[4] Fluent Inc. Lebanon, NH 2002

[5] E. Leonardi, T.A. Kowalewski, V. Timchenko, G. de Vahl Davis "Effects of Finite Wall Conductivity on Flow Structures in Natural Convection”, CHMT99, Cyprus, pp. 182-188, EM University Printinghouse, 1999

[6] H. Sadat and S. Couturier "Performance and Accuracy of a Meshless Method For Laminar Natural Convection" Numerical Heat Transfer, Part B, 37:455-467, 2000

[7] I. Kovacevic, A. Poredos, B. Sarler, "Solving the Stefan problem with the radial basis function collocation method". Numerical Heat Transfer, Part B,44:1-24,2003. 